\section{Lesiones en cavidad oral en pacientes con VIH en un estado del sur de México}

\section{Oral cavity injuries in patients with HIV in a state in southern Mexico}

\begin{abstract}
Resumen
Objetivo: Determinar la frecuencia de lesiones de la cavidad oral en personas portadoras de VIH que son atendidas en la unidad médica "Centro Ambulatorio para la prevención y atención en SIDA e Infecciones de Transmisión Sexual" (CAPASITS) de la ciudad de Tuxtla Gutiérrez, Chiapas; México. Métodos: Se realizó un estudio observacional, transversal y prospectivo en pacientes con diagnóstico reciente de VIH, que incluyó la revisión clínica bucal. Resultados: Se incluyeron a 58 pacientes con diagnóstico de infección por VIH que asistieron al centro en el periodo de junio 2016 a marzo del 2017, siendo 75,9\% hombres, con 31,6 años en promedio, el 55,2\% declaró ser heterosexual y con un nivel de instrucción primaria $(42,9 \%)$. El grupo de patologías bucales con mayor frecuencia encontrada fueron las micóticas $(89,7 \%)$, seguida de las bacterianas $(72,4 \%)$ y virales $(65,5 \%)$; las lesiones más frecuentes fueron la candidiasis pseudomembranosa $(98,1 \%)$, la leucoplasia vellosa $(60,3 \%)$ y el eritema gingival $(56,9 \%)$. Conclusiones: El $77,6 \%$ presentaron de dos a cuatro lesiones, por lo que las lesiones presentes en cavidad oral pueden ser indicadoras del nivel del estado inmunológico de un individuo, por lo tanto, el odontólogo puede ser el primer profesional de salud en sospechar la presencia de la infección por VIH.
\end{abstract}

Palabras clave: Infecciones por VIH; Candidiasis bucal; Leucoplasia bucal; Gingivitis; México.

\begin{abstract}
Objective: To determine the frequency of lesions of the oral cavity in people with HIV who are treated in the medical unit "Ambulatory Center for the prevention and care in AIDS and Sexually Transmitted Infections" (CAPASITS) of the city of Tuxtla Gutiérrez, Chiapas; Mexico. Methods: An observational, cross-sectional and prospective study was conducted in patients with a recent diagnosis of HIV, which included oral clinical review. Results: We included 58 patients diagnosed with HIV infection who were attended in the center in the period from June 2016 to March 2017, 75.9\% were men, with an average of 31.6 years, $55.2 \%$ of them admitted heterosexuality and a primary level education $(42.9 \%)$. The group of oral pathologies most frequently found were mycotic $(89.7 \%)$, followed by bacterial $(72.4 \%)$ and viral $(65.5 \%)$, the most frequent lesions were pseudomembranous candidiasis (98.1\%), hairy leukoplakia (60.3\%) and gingival erythema (56.9\%). Conclusions: $77.6 \%$ of the patients presented from two to four lesions, so that lesions in the oral cavity can be indicative of the level of the immune status of an individual, therefore, the dentist may be the first health professional to suspect the presence of HIV infection.
\end{abstract}

Keywords: HIV infections; Oral candidiasis, Oral leukoplakia, Gingivitis; Mexico.
ISSN: 1560-9111

\section{Artículo Original}

\author{
Josymar Chorley-Sánchez ${ }^{1, a}$, Juan Carlos \\ Nájera-Ortiz ${ }^{2, b}$ \\ ${ }^{1}$ Universidad de Ciencias y Artes de Chiapas \\ Facultad de Ciencias Odontológicas y Salud \\ Pública. Chiapas, México. \\ ${ }^{2}$ Universidad de Ciencias y Artes de Chiapas. \\ Facultad de Ciencias Odontológicas y Salud \\ Pública. Grups de Recerca d' Amèrica i Àfrica \\ Llatines (GRAAL). Chiapas, México. \\ ${ }^{a}$ Maestro en Ciencias. \\ ${ }^{\mathrm{b}}$ Doctor en Ciencias.
}

\author{
Correspondencia: \\ Juan Carlos Nájera Ortiz \\ Correo electrónico: juan.najera@unicach.mx \\ Libramiento Norte Poniente 1150, Colonia \\ Potinaspak, Tuxtla Gutiérrez, Chiapas, México.
}

Coautor:

Josymar Chorley-Sánchez

josymar.chorley@unicach.mx

Conflicto de intereses: Los autores declaran no tener conflictos de interés.

Fuente de financiamiento: Autofinanciado.

Fecha de recepción: 04/10/18

Fecha de aceptación: 21/12/18

( ) Los autores. Este artículo es publicado por la revista Odontología Sanmarquina de la Facultad de Odontología, Universidad Nacional Mayor de San Marcos. Este es un artículo de acceso abierto, distribuido bajo los términos de la licencia Creative Commons Atribucion - No Comercia_Compartir Igual 4.0 Internacional. (http://creativecommons.org/licenses/by-nc-sa/4.0/) que permite el uso no comercial, distribución y reproducción en cualquier medio, siempre que la obra original sea debidamente citada. 


\section{Introducción}

El virus de la inmunodeficiencia humana (VIH) altera o anula las funciones de las células del sistema inmune, principalmente las de tipo $\mathrm{CD} 4{ }^{1}$, en consecuencia, la infección por este virus produce un deterioro progresivo del sistema inmunitario de tipo celular, conduciendo a una "inmunodeficiencia", dejando de cumplir su función de contrarrestar las infecciones y otras enfermedades ${ }^{2}$.

Debido a esta condición de inmunodeficiencia, en los pacientes portadores del VIH pueden presentarse diferentes enfermedades consideradas oportunistas tanto infecciosas (lesiones de etiología viral, bacteriana, fúngica y secundaria a parásitos), como no infecciosas (asociadas al tratamiento antirretroviral de alta calidad -TARAA-, xerosis, dermatitis seborreica, psoriasis, reacciones adversas a medicamentos, neoplasias, entre otras) ${ }^{3}$.

Dentro de las enfermedades o manifestaciones infecciosas; las de tipo bucal y perioral son las más comunes en personas infectadas por el VIH, por lo tanto, es frecuente que puedan usarse como indicadoras y predictivas del deterioro del estado general de la salud, así como de un pronóstico negativo de la misma; además de que puede tener un valor diagnóstico importante al momento de evaluar el estado del sistema Inmune del paciente ${ }^{4,5}$ y por lo tanto, la susceptibilidad de padecer otras infecciones oportunistas. De acuerdo a lo reportado por la literatura, la mayoría de las personas con infección por el VIH, en algún momento o estadio de la enfermedad presentarán alguna manifestación en cabeza y/o cuello ${ }^{6}$, representando las lesiones en la cavidad bucal, signos tempranos de esta infección ${ }^{7}$. No obstante a estas evidencias, en pocas ocasiones se sospecha de que estas lesiones se encuentren relacionadas con la infección por $\mathrm{VIH}^{8}$.

De acuerdo a cifras del Centro Nacional para la Prevención y Control del VIH y el SIDA (CENSIDA) ${ }^{2}$ dependiente de la Secretaría de Salud de México, a finales del 2016, se registró en todo el país un total de 254871 casos notificados de VIH/SIDA, de los cuales, 66327 (26\%), fueron casos registrados como seropositivos a VIH y, 188544 (74\%) como casos de SIDA. En Chiapas en este mismo año se tenía un registro total de 12480 casos de VIH/SIDA, siendo 2681 casos de VIH y 9799 de SIDA .

Es en este sentido, existen regiones en el estado de Chiapas donde debido al alto número de casos reportados resulta importante sospechar que la presencia de algunas de estas lesiones se encuentra fuertemente asociadas a la infección por VIH, como lo son la candidiasis (pseudomembranosa y eritematosa), leucoplasia vellosa, sarcoma de Kaposi, linfoma no Hodgkin y formas especiales de enfermedad periodontal como eritema gingival lineal, gingivitis y periodontitis necrotizante.

Es por ello que el presente estudio, tiene como objetivo determinar la frecuencia de lesiones de la cavidad oral en pacientes con diagnóstico de VIH/SIDA de una unidad de referencia de atención de pacientes portadores del virus en el estado de Chiapas, México.

\section{Métodos}

Se realizó un estudio con diseño observacional de corte transversal que incluyó la revisión clínica de los pacientes con diagnóstico reciente de VIH, ingresados en la unidad médica "Centro Ambulatorio para la Prevención y Atención en SIDA e Infecciones de Transmisión Sexual" (CAPASITS) de Tuxtla Gutiérrez, Chiapas, México en el periodo del 01 de junio del 2016 al 31 de marzo del 2017.

La información socioeconómica y demográfica necesaria para cumplir con los objetivos del presente estudio fue tomada de los expedientes clínicos que la Unidad Médica "CAPASITS", los cuales, a la vez están diseñados de acuerdo a lo estipulado por la normativa vigente en México ${ }^{10}$. Por su parte, la revisión clínica odontológica fue llevada a cabo por un odontólogo previamente capacitado, cumpliendo con lo establecido en la Guía para la Atención Estomatológica en Personas con VIH ${ }^{7}$. La revisión de la cavidad bucal de estas personas se encaminó hacia la búsqueda intencionada de infecciones y enfermedades vinculadas con la inmunosupresión (eritema gingival lineal, gingivitis necrotizante, periodontitis necrotizante, candidiasis pseudomembranosa, candidiasis eritematosa, candidiasis hiperplásica, queilitis angular, leucoplasia vellosa, infección por virus del herpes simple, estomatitis aftosa recurrentes, infección por virus del papiloma humano, sarcoma de Kaposi, linfoma no Hodgkin), por lo que el diagnóstico de las patologías para cada caso se llevó a cabo clínicamente mediante la presencia de lesiones características visibles.

Para la descripción de las variables incluidas en el estudio, se emplearon recursos de estadística univariada, tales como frecuencias absolutas y relativas, además de medidas de tendencia central.

El protocolo del estudio fue aprobado por el Comité de Ética del CAPACITS, además de que todos los procedimientos fueron adecuados a las recomendaciones para la investigación biomédica de Helsinki de la Asociación Médica Mundial ${ }^{11}$ y a la Ley General de Salud de Méxi$\mathrm{Co}^{12}$. Este estudio es de riesgo mínimo, como lo estipula la mencionada ley.

\section{Resultados}

Descripción sociodemográfica de los pacientes en estudio. En el presente estudio se incluyeron un total de 58 pacientes con diagnóstico reciente de infección por VIH que fueron atendidos durante el periodo marcado. El promedio de edad de este grupo de personas fue de 31,6 ańos $(\mathrm{DE}=9,9)$, es por ello que, el $48,2 \%$ del total de pacientes, se encontraba en el grupo de edad entre 25 y 34 años, en cuanto al sexo, 44 fueron hombres $(75,9$ \%) (Tabla 1).

Al revisar la distribución de la población según la preferencia sexual, poco más de la mitad refirió ser heterosexual $(55,2 \%)$, asimismo, respecto al estado civil, se encontró que la mayoría eran solteros $(57,9 \%)$ (Tabla 1 ). 
Una gran proporción de las personas participantes en el presente estudio refirieron haber cursado cuando mucho la instrucción primaria $(42,9 \%)$ y $39(67,2 \%)$ tuvo una ocupación remunerada. En cuanto a la religión que profesan los pacientes, más de la mitad mencionó ser católico $(61,4 \%)$ (Tabla 1$)$.

Tabla 1. Características sociodemográficas de los pacientes con diagnóstico de VIH/SIDA

\begin{tabular}{|c|c|c|c|}
\hline \multicolumn{2}{|c|}{ Variables Sociodemográficas } & \multirow{2}{*}{$\begin{array}{l}\mathbf{n} \\
44\end{array}$} & \multirow{2}{*}{\begin{tabular}{|c}
$\mathbf{\%}$ \\
75,9
\end{tabular}} \\
\hline Sexo & Masculino & & \\
\hline & Femenino & 14 & 24,1 \\
\hline \multirow[t]{5}{*}{ Grupo de edad (años) } & $18-24$ & 13 & 22,4 \\
\hline & $25-29$ & 14 & 24,1 \\
\hline & $30-34$ & 14 & 24,1 \\
\hline & $35-39$ & 11 & 19,0 \\
\hline & 40 y más & 6 & 10,3 \\
\hline \multirow[t]{3}{*}{ Preferencia sexual } & Heterosexual & 32 & 55,2 \\
\hline & Homosexual & 20 & 34,5 \\
\hline & Bisexual & 6 & 10,3 \\
\hline \multirow[t]{4}{*}{ Estado civil } & Soltero & 33 & 57,9 \\
\hline & Casado & 18 & 31,0 \\
\hline & Divorciado & 3 & 5,3 \\
\hline & Viudo & 3 & 5,3 \\
\hline \multirow[t]{5}{*}{ Grado escolar } & Ninguna & 7 & 12,5 \\
\hline & Primaria & 17 & 30,4 \\
\hline & Secundaria & 6 & 10,7 \\
\hline & Preparatoria & 13 & 23,2 \\
\hline & Licenciatura & 13 & 23,2 \\
\hline \multirow[t]{4}{*}{ Ocupación } & Desempleado & 6 & 10,3 \\
\hline & Trabaja & 39 & 67,2 \\
\hline & Ama de casa & 10 & 17,2 \\
\hline & Estudiante & 3 & 5,2 \\
\hline \multirow[t]{3}{*}{ Religión } & Ninguna & 12 & 21,1 \\
\hline & Católica & 35 & 61,4 \\
\hline & Diferente a católica & 10 & 17,5 \\
\hline
\end{tabular}

Fuente: Expedientes clínicos del CAPACITS

Frecuencia de lesiones de la cavidad oral en los pacientes estudiados. En cuanto a la presencia de lesiones bucales, de forma general, todos los pacientes $(n=58)$ posterior a la revisión clínica odontológica presentaron al menos una lesión en la cavidad oral, de los cuales $6,9 \%$ presentó solamente una lesión y el 77,6\% presentó entre 2 y 4 lesiones. Por grupo de lesiones de acuerdo a su naturaleza, $42(72,4 \%)$ de los usuarios presentó alguna lesión de tipo bacteriana, $52(89,7 \%)$ de tipo micótica y $38(65,5 \%)$ de tipo viral, no se tuvo registró de patologías neoplásicas (Tabla 2).

En este sentido, dentro de las lesiones bacterianas diagnosticadas clínicamente $(\mathrm{n}=42)$, el eritema gingival lineal fue el más frecuente $(78,6 \%)$ para este grupo (Figura 1), la candidiasis pseudomembranosa $(98,1 \%)$ (Figura 2) y la leucoplasia vellosa (92,1\%) (Figura 3) respectivamente para las lesiones de etiología micótica $(n=52)$ y viral $(n=38)$ (Tabla 3), en este sentido, fue la candidiasis pseudomembranosa la patología que presentó mayor frecuencia $(87,9 \%)$.

Tabla 2. Presencia del número y tipo de lesiones en pacientes con diagnóstico de VIH/SIDA

\begin{tabular}{lcc}
\hline Lesiones en la cavidad bucal & $\mathbf{n}$ & $\mathbf{\%}$ \\
\hline Número de lesiones & & \\
Una lesión & 4 & 6,9 \\
2-4 lesiones & 45 & 77,6 \\
5 y más lesiones & 9 & 15,5 \\
\hline Tipo de lesiones & & \\
Con lesiones de tipo bacteriana & 42 & 72,4 \\
Con lesiones de tipo micótica & 52 & 89,7 \\
Con lesiones de tipo viral & 38 & 65,5 \\
\hline
\end{tabular}

Fuente: Expedientes clínicos del CAPACITS

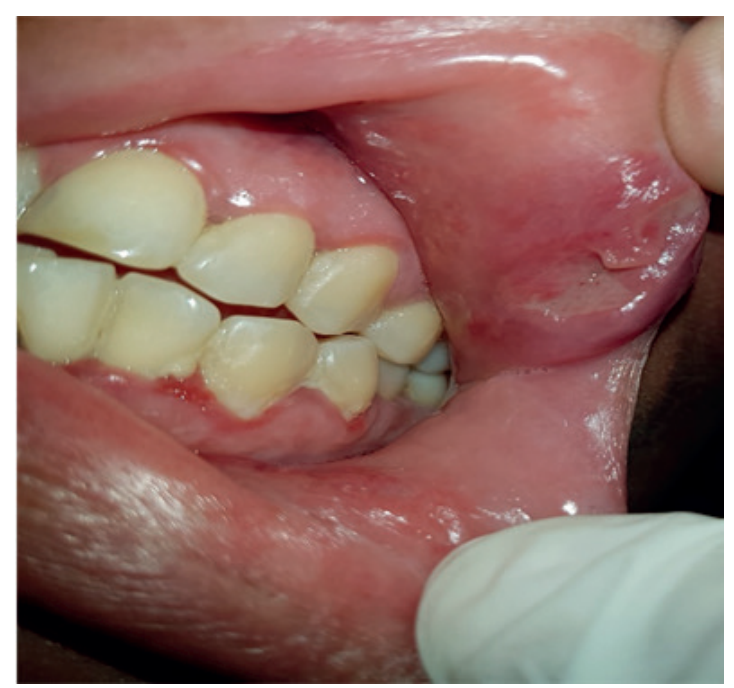

Figura 1. Paciente con lesión bacteriana eritema gingival lineal, localizada en el incisivo lateral, canino y premolar izquierdo (piezas $32,33,34$ ). Lesión viral estomatitis aftosa recurrente de tipo menor y tipo profunda con forma irregular en labio superior

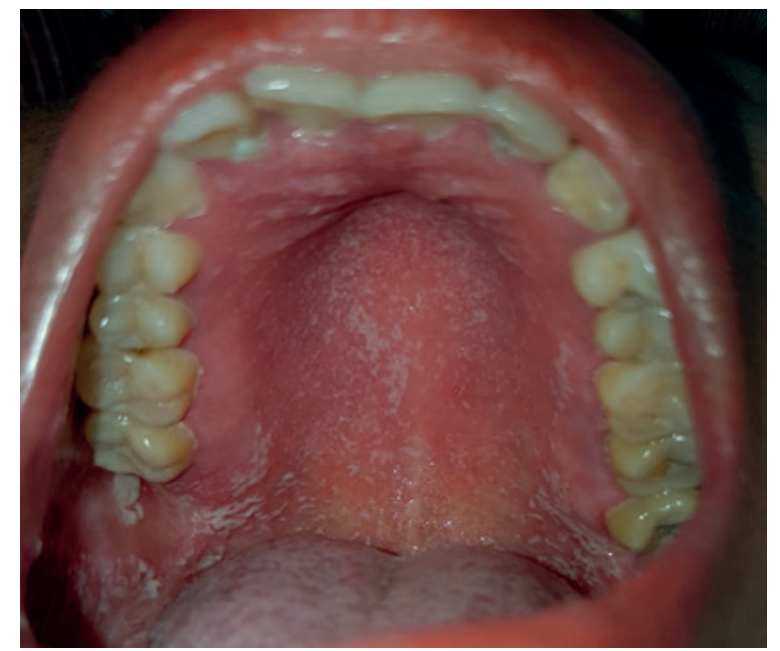

Figura 2. Paciente con lesión micótica candidiasis pseudomembranosa en forma de placas aterciopeladas y de color blanco, blandas, cremosas, semiadherentes (tipo muguet) con interposicion de zonas eritematosas en la región del paladar 


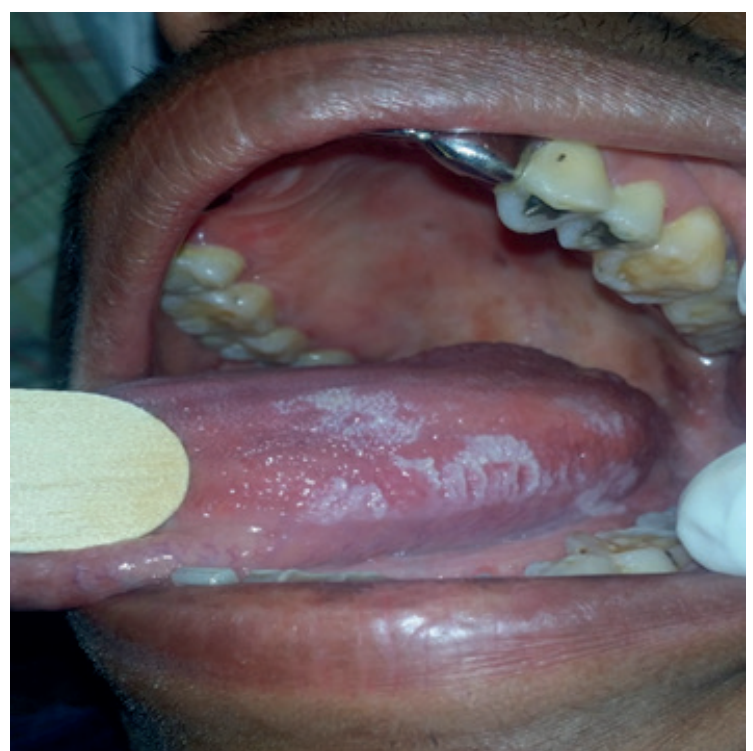

Figura 3. Paciente con lesión viral leucoplasia vellosa, en forma de placas blancas no removibles, bien delimitadas, de aspecto filiforme y corrugado situada en el borde lateral de la lengua

Tabla 3. Frecuencia de lesiones en la cavidad bucal en pacientes con diagnóstico de VIH/SIDA

\begin{tabular}{lccc}
\hline Patologías Bucales & n & \% & $\begin{array}{c}\text { Prevalencia } \\
\mathbf{n = 5 8}\end{array}$ \\
\hline Bacterianas & $\mathbf{4 2}$ & $\mathbf{1 0 0}$ & $\mathbf{7 2 , 4}$ \\
Eritema gingival lineal & 33 & 78,6 & 56,9 \\
Gingivitis necrotizante & 17 & 40,5 & 29,3 \\
Periodontitis necrotizante & 4 & 9,5 & 6,9 \\
\hline Micóticas & 52 & $\mathbf{1 0 0}$ & $\mathbf{8 9 . 7}$ \\
Candidiasis pseudomembranosa & 51 & 98,1 & 87.9 \\
Candidiasis eritematosa & 19 & 36,5 & 32.7 \\
Candidiasis hiperplásica & 8 & 15,4 & 13.8 \\
Queilitis angular & 17 & 32,7 & 29.3 \\
\hline Virales & 38 & $\mathbf{1 0 0}$ & $\mathbf{6 5 . 5}$ \\
Leucoplasia vellosa & 35 & 92,1 & 60.3 \\
Infección por Virus del herpes & 3 & 7,9 & 5.2 \\
simple & & & 5.2 \\
$\begin{array}{l}\text { Estomatitis aftosa recurrente de tipo } \\
\text { menor }\end{array}$ & 3 & 7,9 & 3.4 \\
$\begin{array}{l}\text { Infección por Virus del papiloma } \\
\text { humano }\end{array}$ & 2 & 5,3 & \\
\hline
\end{tabular}

Fuente: Expedientes clínicos del CAPACITS

\section{Discusión}

La frecuencia de la infección por VIH en relación al sexo muestra un predominio en el sexo masculino en un $75,9 \%$, correspondiendo a lo reportado por el Registro Nacional para la Prevención y Control del VIH/SIDA ${ }^{13}$ en el 2016 para el estado de Chiapas en México, que fue del $73 \%$. Es importante considerar que si bien, las personas participantes en el estudio no provienen de una muestra probabilística, el CAPACITS es un centro de referencia para la atención de personas portadoras del VIH de todo el estado, por lo que puede acercarse mucho a la distribución de la población con esta infección.
El resto de las variables sociodemográficas de la población participante del estudio demostraron que se trata de un grupo de personas jóvenes, ya que el $70,6 \%$ tenía entre 18 y 34 años (edad media: 31,6 años), que el $67,2 \%$ se encuentra ocupado de manera remunerada, aunque del sector primario (albañil, comerciante), lo que coincide con el grado de escolaridad declarado (43\% había cursado como mucho la instrucción primaria). Aunado a esto, son solteros el $58 \%$, poco más de la mitad menciono ser heterosexual y con religión católica el $61,4 \%$.

Aunque para el presente estudio sólo se consideraron personas mayores de 18 años, existen evidencias donde se menciona que, entre la población general seropositiva (incluyendo niños), se presentan de manera frecuente lesiones en la cavidad oral ${ }^{14}$. En relación a esta situación, es de resaltar que el 100\% los pacientes VIH-positivos participantes de nuestra investigación, se les fue diagnosticada al menos una alteración en el sistema estomatognático tras llevar a cabo una revisión clínica odontológica.

Otros autores han marcado la relación entre el estado de salud bucal tanto con la presencia de la infección por VIH, como con el avance de la misma, por ejemplo, se han reportado alteraciones en cabeza y cuello en más del 95\% de pacientes seropositivos, dependiendo del avance de la enfermedad ${ }^{15}$, de esta manera, se han documentado lesiones en cavidad oral de un $50-80 \%$ en personas VIH-positivos y más de $80 \%$ en presencia de SIDA ${ }^{16,17}$.

En relación a lo antes citado, se han agrupado a más de 40 manifestaciones bucales asociadas en diferentes grados con la infección por VIH, para este estudio en particular, los pacientes que acudieron a consultarse a este Centro de referencia solo presentaron 11 de estas, correspondiendo a las más frecuentes. De estas, tomando la clasificación y diagnóstico para las lesiones orales en la infección por VIH ${ }^{18}$, donde en el grupo 1 considera a las lesiones estrechamente relacionadas con la infección VIH, se encontró la presencia del eritema gingival lineal conocida también con el nombre de gingivitis asociada al VIH o gingivitis de banda roja, la gingivitis necrotizante, la periodontitis necrotizante, la candidiasis pseudomembranosa, la candidiasis eritematosa, la candidiasis hiperplásica, la queilitis angular y la leucoplasia vellosa. Del grupo 2, que incluye lesiones menos comúnmente asociadas a la infección VIH, el virus del herpes simple, la estomatitis aftosa recurrente y el virus del papiloma humano.

La candidiasis bucal (pseudomembranosa) fue la patología más frecuente para el presente estudio, es también la más fuertemente asociada a la infección por el VIH, por lo que se constituye como una alteración con alta prevalencia en personas seropositivas. Se estima un aumento en el riesgo de presentarla entre el 25 a 50 veces más cuando se es de esta condición serológica, además de que los episodios son recurrentes, con un incremento en la frecuencia y severidad con la progresión de la enfermedad ${ }^{15,16}$. Se ha observado la presencia de candidiasis cuando hay un deterioro inmunológico importante, es 
decir, al disminuir los linfocitos CD4+ a menos de 400 por $\mathrm{mm}^{3}$ en sangre, o puede ser la primera expresión clínica al adquirirse una infección por VIH ${ }^{14,17}$.

En cuanto a la queilitis angular, es considerada la tercera forma más frecuente de candidiasis en las personas VIH positivos, seguida de la pseudomembranosa y la eritematosa ${ }^{19}$, lo cual es consistente con lo encontrado en este estudio, ya que fue diagnosticada en una tercera parte de las personas participantes en este estudio.

Las úlceras tipo afta se presentaron con una prevalencia de 7,9\% entre las lesiones de tipo viral, resultado que concuerda con lo declarado por Rioboo y Bascones ${ }^{20}$ donde mencionan dentro de la etiogenia de estas lesiones una posible desproporción de la relación de los linfocitos CD4 y CD8, presentándose cuentas de menos de $200 \mathrm{CD} 4+$ por mililitro, tal y como sucede en muchos casos de personas con VIH recién diagnosticadas. Santos y colaboradores ${ }^{21}$ resaltan que los pacientes no recuerdan haber presentado episodios de aftas recurrentes antes de ser portadores del VIH, además que el número de lesiones por episodio es mayor cuando avanzan a SIDA, por lo que también se puede considerar un indicador.

Por su parte, la leucoplasia vellosa, considerada también un indicio temprano de inmunosupresión, en relación a una infección por VIH de una carga viral elevada independiente del conteo de células T CD4 + y de progresión de la infección, con un alto valor predictivo, el 95\% de las leucoplasias vellosas se encuentran asociadas con el VIH ${ }^{22,23}$. Es en este sentido que Pinzón y colaboradores (2008) ${ }^{24,25}$ desarrollaron un estudio en Cali, Colombia y encontraron una prevalencia del $13,6 \%$, donde refieren que en África oscila entre 0\% y 20\%, en Asia de 3\% a 13\%, en Estados Unidos y Europa entre 7\% y 30\% y México del $43 \%$, cuando en nuestra población correspondió al 60\% dentro de las enfermedades diagnosticadas en el sistema estomatognático. La relevancia de la leucoplasia vellosa está relacionada al igual que otras lesiones en la cavidad bucal, con su valor predictivo ${ }^{22}$.

De esta manera y de acuerdo a estudios ya publicados 6,17,24, las lesiones en la cavidad oral se presentarán tanto en pacientes infectados por el VIH bajo un control médico, como sin él, por lo que, se podrían realizar futuros estudios donde se revisen prevalencias y factores que se relacionen con la presencia de manifestaciones bucales en pacientes que acudan al CAPASITS y que estén bajo control médico, corroborando de esta manera si los resultados son similares a los que se han publicado en pacientes que reciben tratamiento antirretroviral.

De acuerdo a lo reportado por diversos autores, en los países con pocos recursos económicos, la prevalencia de lesiones orales varía de una región a otra, por lo tanto, la diferencia entre resultados de las investigaciones realizadas en varias partes del mundo es frecuente, tal y como pudo corroborarse con los resultados del presente estudio, como con los ya publicados ${ }^{26-30}$. En consecuencia, no hay consenso sobre la magnitud de la prevalencia de estas lesiones orales en pacientes infectados por el VIH, posiblemente por la influencia de las características sociodemográficas y clínicas; propias de cada persona y por las particularidades de cada grupo poblacional ${ }^{31,32}$.

Este estudio constituye un punto de partida para futuras investigaciones, describe de manera general las características clínicas y sociodemográficas de pacientes con diagnóstico reciente de VIH en un estado del sur de México. Conforme se realicen más estudios, se tendrá una mejor aproximación a la realidad y servirá para fortalecer actividades de diagnóstico, tratamiento y prevención en la práctica profesional, promoviendo el autocuidado, el autoexamen bucal y la búsqueda de tratamiento odontológico oportuno. Pese a que la muestra de estudio es pequeña y el tiempo de estudio fue corto, aun así, permite hacer una valoración general del comportamiento actual de estas lesiones bucales en los pacientes afectados teniendo congruencia con los resultados obtenidos y los que se reportan en la literatura mundial.

\section{Referencias bibliográfícas}

1. Organización Mundial de la Salud (OMS) [Internet]. Organización Mundial de la Salud (OMS)/SIDA. Ginebra, Suiza 2017. Fecha de consulta: 15 de septiembre de 2018. Disponible en http://www.who.int/topics/ hiv_aids/es/.

2. CENSIDA. [Internet]. Guía integral para personas recién diagnosticadas con VIH. Primera edición, México, D.F. 2016. Fecha de consulta: 18 de septiembre de 2018. Disponible en http://www.censida.salud.gob.mx/interior/guiasmanuales.html.

3. Garza GR, González GSE, Ocampo CJ. Manifestaciones cutáneas del VIH. Gac Med Mex. 2014;150(s2):194221.

4. Reichart PA, Khongkhunthian P, Bendick C. Oral manifestations in HIV infected individuals from Thailand and Cambodia. Med Microbiol Immunol. 2003;192:157-60.

5. Ranganathan $K$, Umadevi $M$, Saraswathi TR, Kumarasamy N, Solomon S, Johnson N. Oral lesions and conditions associated with Human Immunodeficiency Virus infection in 1000 South Indian Patients. Ann Acad Med Singapore. 2004;33:37-42.

6. Centro Nacional de Programas Preventivos y Control de Enfermedades (CENAPRECE), Centro Nacional para la Prevención y el Control del VIH y el sida (CENSIDA). Guía para la atención Estomatológica en Personas con VIH, México 2015. Primera Edición 2015

7. Ruiz P, Rodriguez A, Luengas E, Ramos U,Cruz J,Cruz M. Guía para la Atención Estomatológica en Personas con VIH. 1a ed. México. 2015; ISBN: 978-607-460477-1. Disponible en: http://www.censida.salud.gob. $\mathrm{mx} /$ descargas/biblioteca/GUIA_ESTOMATOLOGICA2015.pdf

8. Pinheiro A, Marcenes W, Zakrzewska JM, Robinson P. Dental and Oral lesions in HIV infected patients: a study in Brazil. Int Dent J. 2004;54:131-137.

9. Coogan MM, Greenspan J, Challacombe SJ. Oral lesions in infection with human immunodeficiency virus. Bull World Health Organ. 2005;83(9):700-706 
10. CENSIDA. [Internet]. Vigilancia Epidemiológica de casos de VIH/SIDA en México, Registro Nacional de Casos de SIDA, Actualización al Cierre 2016. México, D.F. 2017. Fecha de consulta: 18 de septiembre de 2018. Disponible en http:/www.gob.mx/censida/documentos/ epidemiologia-registro-nacional-de-casos-de-sida

11. Secretaría de Salud (SSA). Modificación a la Norma Oficial Mexicana NOM-010-SSA2-1993, Para la prevención y control de la infección por Virus de la Inmunodeficiencia Humana. Diario Oficial de la Federación 21 de junio del año 2000. Disponible en http://www.salud. gob.mx/unidades/cdi/nom/m010ssa23.html

12. World Medical Association (WMA). Declaration of Helsinki: Ethical Principles for Medical Research Involving Human Subjects . Edinburgh: WMA, 2000.

13. Secretaría de Salud (SSA). Ley General de Salud Mexico y disposiciones complementarias. Ciudad de México, México: Porrúa, 1994.

14. SSA, Dirección General de Epidemiología (DGE), CENSIDA. Vigilancia Epidemiológica de casos de VIH/ SIDA en México Registro Nacional de Casos de SIDA. Actualización al Cierre del 2016. Disponible en https:// www.gob.mx/cms/uploads/attachment/file/218474/ RN_Cierre_2016.pdf

15. Estrada-Montojo JH, Escobar-Lepuizomo, DN. Manifestaciones orales de la infección por VIH/sida en niños y adolescentes: aspectos clínicos, epidemiologicos y pautas de tratamiento. Univ Odontol. 2011;30(64):37-50.

16. Bascones A. Serrano C, Campos J. Manifestaciones de la infección por el virus de la inmunodeficiencia humana en la cavidad bucal. Med Clin. 2003 (Barc); 120(11):426-34.

17. Coogan M, Greenspan J, Chollacombe S. Oral lesions in infection with human immunodeficiency virus. Bull WHO. 2005;83:700-706.

18. Bravo IM, Correnti M, Escalona L, Perrone M, Brito A, Tovar V, et al. Prevalence of oral lesions in HIV patients related to CD4 cell count and viral load in o Venezuelan population. Med Oral Patol Oral Cir Bucal. 2006;11:E33- E39.

19. EC Clearinghouse on Oral Problems Related to HIV Infection and WHO Collaborating Centre on Oral Manifestations of the Human Immunodeficiency Virus. Classification and diagnostic criteria for oral lesions in HIV infection. Journal of Oral Pathology and Medicine. 1993;22:289-91.

20. Aguirre Urízor JM, Echebarría-Goicourío MA, Eguío del Valle A. Síndrome de inmunodeficiencia adquirida: manifestaciones en la cavidad bucal. Med Oral Patol Oral Cir Bucal. 2004; 9 suppI; S148-157.

21. Rioboo-Crespo M, Bascones-Martínez A. Aftas de la mucosa oral. Av Odontoestomatol. 2011;27(2):63-74.

22. Santos-Pinheiro R, Ribeiro de Fronça T, Rocha B, Carvalho-Ferreira D, Beder-Ribeiro CM, Beota-Covalcanti SM, et al. Human papilloma - virus coinfection in the oral cavity of HIV- infected children. J Clin Pathol. 2011;64:1083-1087.

23. González X, Correnti M, Rivera H, Perrone M. Detección de Candida albicans en lesiones de leucoplasia vellosa bucal de un grupo de pacientes venezolanos VIH+. Acta Odontol Venez. 2010;48(2):1-10.

24. Calzada-Martínez B, Gallegos-Rodríguez CR. Manifestaciones bucales en reclusos portadores de VIH/SIDA en la unidad especial SIDA. Rev cienc méd La Habana. 2011;17(2):226-232.

25. Pinzón EM, Bravo SM, Méndez F, Clavijo GM, León ME. Prevalencia y factores relacionados con la presencia de manifestaciones orales en pacientes con VIH/SIDA, Cali, Colombia. Colomb Med. 2008;39(4):346-55.

26. Campo J, Del romero J, Castilla J, García S, Rodríguez $\mathrm{C}$, Bascones A. Oral candidiasis as a clinical marker related to viral load, CD4 lymphocyte count and CD4 Lymphocyte percentage in HIV-infected patients. J Oral Pathol Med. 2002;31(1):5-10.

27. Campisi G, Pizzo G, Mancuso S, Margiotta V. Gender diferences in human immunodeficiency virus-related oral lesions: an Italian study. Oral Surg Oral Med Oral Pathol Oral Radiol Endod. 2001;91(5):546-51.

28. Bendick C, Scheifele C, Reichart PA. Oral manifestations in 101 Cambodian patients with HIV infection and AIDS. J Oral Pathol Med. 2002;31(1):1-4.

29. Patton LL, Phelan JA, Ramos-Gómez FJ, Nittayananta W, Shiboski CH, Mbuguye TL. Prevalence and classification of HIV-associated oral lesions. Oral Dis. 2002;8 Suppl 2:98-109.

30. Tovar V, Guerra M, Bravo I, Albornoz E. Manifestaciones bucales e infecciones oportunistas más frecuentes encontradas en 208 pacientes con infección por $\mathrm{VIH} /$ sida. Acta Odontol Venez. 2002;40(3):260-264.

31. Singh A, Bairy I, Shivananda PG. Spectrum of op- portunistic infections in AIDS cases. Indian J Med Sci. 2003;57(1):16-21.

32. Benito-Urdaneta M, Benito-Urdaneta M, Bernardoni de Socorro C, Arteaga de Vizcaíno M, Sotolongo M, Benito MT, et al. Manifestaciones bucales en pacientes VIH positivos y su relación con valores de linfocitos CD4. Acta Odontol Venez. 2007;45(2):229-33. 